\title{
Delamination Phenomenon in Composite Laminated Plates and Beams
}

\author{
Osama Mohammed Elmardi Suleiman Khayal \\ Department of Mechanical Engineering, Faculty of Engineering and Technology, Nile Valley University, Atbara, Sudan \\ Email address: \\ osamamm64@gmail.com

\section{To cite this article:} \\ Osama Mohammed Elmardi Suleiman Khayal. Delamination Phenomenon in Composite Laminated Plates and Beams. Bioprocess \\ Engineering. Vol. 4, No. 1, 2020, pp. 9-16. doi: 10.11648/j.be.20200401.12
}

Received: December 8, 2019; Accepted: January 13, 2020; Published: February 4, 2020

\begin{abstract}
Failure analysis of laminated composite decks structures has attracted a great deal of interest in recent years due to the increased application of composite materials in a wide range of high-performance structures. Intensive experimental and theoretical studies of failure analysis and prediction are being reviewed. Delamination, the separation of two adjacent plies in composite laminates, represents one of the most critical failure modes in composite laminates. In fact, it is an essential issue in the evaluation of composite laminates for durability and damage tolerance. Thus, broken fibers, delaminated regions, cracks in the matrix material, as well as holes, foreign inclusions and small voids constitute material and structural imperfections that can exist in composite structures. Imperfections have always existed and their effect on the structural response of a system has been very significant in many cases. These imperfections can be classified into two broad categories: initial geometrical imperfections and material or constructional imperfections. Delamination is a critical failure mode in fiber-reinforced composite decks plates and beams. It may lead directly to through-thickness failure owing to interlaminar stresses caused by out of plane loading, curved or tapered geometry, or discontinuities owing to cracks, ply drops or free edges. Impact loading causes multiple delaminations, which can propagate in conjunction with sub laminate buckling, greatly reducing the residual compressive strength.
\end{abstract}

Keywords: Delamination, Composite Laminates, Rectangular Plates, Review

\section{Introduction}

Composites were first considered as structural materials a little more than three quarters of a century ago. From that time to now, they have received increasing attention in all aspects of material science, manufacturing technology, and theoretical analysis.

The term composite could mean almost anything if taken at face value, since all materials are composites of dissimilar subunits if examined at close enough details. But in modern materials engineering, the term usually refers to a matrix material that is reinforced with fibers. For instance, the term "FRP" which refers to Fiber Reinforced Plastic usually indicates a thermosetting polyester matrix containing glass fibers, and this particular composite has the lion's share of today commercial market.

Many composites used today are at the leading edge of materials technology, with performance and costs appropriate to ultra-demanding applications such as space crafts. But heterogeneous materials combining the best aspects of dissimilar constituents have been used by nature for millions of years. Ancient societies, imitating nature, used this approach as well: The book of Exodus speaks of using straw to reinforce mud in brick making, without which the bricks would have almost no strength. Here in Sudan, people from ancient times dated back to Meroe civilization, and up to now used zibala (i.e. animals' dung) mixed with mud as a strong building material.

As seen in Table 1 below, which is cited by David Roylance [1], Stephen et al. [2] and Turvey et al. [3], the fibers used in modern composites have strengths and stiffnesses far above those of traditional structural materials. The high strengths of the glass fibers are due to processing that avoids the internal or external textures flaws which normally weaken glass, and the strength and stiffness of polymeric aramid fiber is a consequence of the nearly perfect alignment of the molecular chains with the fiber axis. 
Table 1. Properties of composite reinforcing fibers.

\begin{tabular}{|c|c|c|c|c|}
\hline Material & $\mathrm{E}\left(\mathrm{GN} / \mathrm{m}^{2}\right)$ & & $\sigma_{b}\left(G N / m^{2}\right)$ & $\varepsilon_{b} \quad(\%)$ \\
\hline E-glass & 72.4 & & 2.4 & 2.6 \\
\hline S-glass & 85.5 & & 4.5 & 2.0 \\
\hline Aramid & 124 & & 3.6 & 2.3 \\
\hline Boron & 400 & & 3.5 & 1.0 \\
\hline H S graphite & 253 & & 4.5 & 1.1 \\
\hline H M graphite & 520 & & 2.4 & 0.6 \\
\hline Material & $\rho\left(\mathrm{Mg} / \mathrm{m}^{3}\right)$ & $E / \rho$ & (MN.m/kg) & $\begin{array}{l}\sigma_{b} / \rho \\
(\mathrm{MN} . \mathrm{m} / \mathrm{kg})\end{array}$ \\
\hline E-glass & 2.54 & 28.5 & & 0.95 \\
\hline S-glass & 2.49 & 34.3 & & 1.8 \\
\hline Aramid & 1.45 & 86 & & 2.5 \\
\hline Boron & 2.45 & 163 & & 1.43 \\
\hline H S graphite & 1.80 & 140 & & 2.5 \\
\hline H M graphite & 1.85 & 281 & & 1.3 \\
\hline
\end{tabular}

Where $\mathrm{E}$ is Young's modulus, $\sigma_{b}$ is the breaking stress, $\varepsilon_{b}$ is the breaking strain, and $\rho$ is the mass density.

These materials are not generally usable as fibers alone, and typically they are impregnated by a matrix material that acts to transfer loads to the fibers, and also to protect the fibers from abrasion and environmental attack. The matrix dilutes the properties to some degree, but even so very high specific (weight-adjusted) properties are available from these materials. Polymers are much more commonly used, with unsaturated styrene-hardened polyesters having the majority of low to medium performance applications and Epoxy or more sophisticated thermosets having the higher end of the market. Thermoplastic matrix composites are increasingly attractive materials, with processing difficulties being perhaps their principal limitation.

Recently, composite materials are increasingly used in many mechanical, civil, and aerospace engineering applications due to two desirable features: the first one is their high specific stiffness (i.e. stiffness per unit density) and high specific strength (i.e. strength per unit density), and the second is their properties that can be tailored through variation of the fiber orientation and stacking sequence which gives the designers a wide spectrum of flexibility. The incorporation of high strength, high modulus and low-density filaments in a low strength and a low modulus matrix material is known to result in a structural composite material with a high strength to weight ratio. Thus, the potential of a two-material composite for use in aerospace, under-water, and automotive structures has stimulated considerable research activities in the theoretical prediction of the behavior of these materials. One commonly used composite structure consists of many layers bonded one on top of another to form a high-strength laminated composite plate. Each lamina is fiber reinforced along a single direction, with adjacent layers usually having different filament orientations. For these reasons, composites are continuing to replace other materials used in structures such as conventional materials. In fact, composites are the potential structural materials of the future as their cost continues to decrease due to the continuous improvements in production techniques and the expanding rate of sales.

\subsection{Structure of Composites}

There are many situations in engineering where no single material will be suitable to meet a particular design requirement. However, two materials in combination may possess the desired properties and provide a feasible solution to the materials selection problem. A composite can be defined as a material that is composed of two or more distinct phases, usually a reinforced material supported in a compatible matrix, assembled in prescribed amounts to achieve specific physical and chemical properties.

In order to classify and characterize composite materials, distinction between the following two types is commonly accepted; see Vernon [4], Jan Stegmann and Erik Lund [5], and David Roylance [1].

1. Fibrous composite materials: Which are composed of high strength fibers embedded in a matrix. The functions of the matrix are to bond the fibers together to protect them from damage, and to transmit the load from one fiber to another. \{See Figure 1\}.

2. Particulate composite materials: These are composed of particles encased within a tough matrix, e.g. powders or particles in a matrix like ceramics.

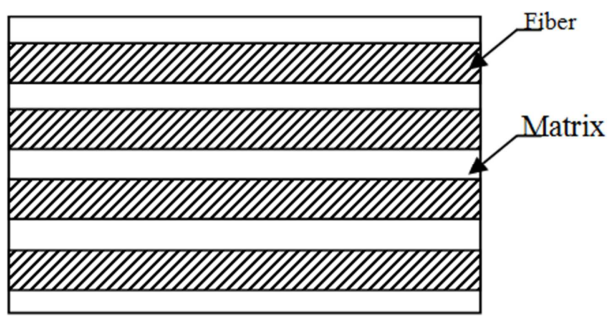

Figure 1. Structure of a fibrous composite.

In this study the focus will be on fiber reinforced composite materials, as they are the basic building element of a rectangular laminated plate structure. Typically, such a material consists of stacks of bonded-together layers (i.e. laminas or plies) made from fiber reinforced material. The layers will often be oriented in different directions to provide specific and directed strengths and stiffnesses of the laminate. Thus, the strengths and stiffnesses of the laminated fiber reinforced composite material can be tailored to the specific design requirements of the structural element being built.

\subsection{Mechanical Properties of a Fiber Reinforced Lamina}

Composite materials have many mechanical characteristics, which are different from those of conventional engineering materials such as metals. More precisely, composite materials are often both inhomogeneous and non-isotropic. Therefore, and due to the inherent heterogeneous nature of composite materials, they can be studied from a micromechanical or a macro mechanical point of view. In micromechanics, the behavior of the inhomogeneous lamina is defined in terms of the constituent materials; whereas in macro mechanics the material is presumed homogeneous and the effects of the constituent materials are detected only as averaged apparent macroscopic properties of the composite material. This 
approach is generally accepted when modeling gross response of composite structures. The micromechanics approach is more convenient for the analysis of the composite material because it studies the volumetric percentages of the constituent materials for the desired lamina stiffnesses and strengths, i.e. the aim of micromechanics is to determine the moduli of elasticity and strength of a lamina in terms of the moduli of elasticity, and volumetric percentage of the fibers and the matrix. To explain further, both the fibers and the matrix are assumed homogeneous, isotropic and linearly elastic.

Stiffness and Strength of a Lamina

The fibers may be oriented randomly within the material, but it is also possible to arrange for them to be oriented preferentially in the direction expected to have the highest stresses. Such a material is said to be anisotropic (i.e. different properties in different directions), and control of the anisotropy is an important means of optimizing the material for specific applications. At a microscopic level, the properties of these composites are determined by the orientation and distribution of the fibers, as well as by the properties of the fiber and matrix materials.

Consider a typical region of material of unit dimensions, containing a volume fraction, $\mathrm{Vf}$ of fibers all oriented in a single direction. The matrix volume fraction is then, $V_{m}=1-V_{f}$. This region can be idealized by gathering all the fibers together, leaving the matrix to occupy the remaining volume. If a stress $\sigma_{l}$ is applied along the fiber direction, the fiber and matrix phases act in parallel to support the load. In these parallel connections the strains in each phase must be the same, so the strain $\varepsilon_{l}$ in the fiber direction can be written as:

$$
\varepsilon_{l}=\varepsilon_{f}=\varepsilon_{m}
$$

(Where: the subscripts $1, \mathrm{f}$ and $\mathrm{m}$ denote the lamina, fibers and matrix respectively).

The forces in each phase must add to balance the total load on the material. Since the forces in each phase are the phase stresses times the area (here numerically equal to the volume fraction), we have

$$
\sigma_{l}=\sigma_{f} V_{f}+\sigma_{m} V_{m}=E_{f} \varepsilon_{l} V_{f}+E_{m} \varepsilon_{l} V_{m}
$$

The stiffness in the fiber direction is found by dividing the stress by the strain:

$$
E_{l}=\frac{\sigma_{l}}{\varepsilon_{l}}=E_{f} V_{f}+E_{m} V_{m}
$$

(Where: $\mathrm{E}$ is the longitudinal Young's modulus)

This relation is known as a rule of mixtures prediction of the overall modulus in terms of the moduli of the constituent phases and their volume fractions.

Rule of mixtures estimates for strength proceed along lines similar to those for stiffness. For instance, consider a unidirectional reinforced composite that is strained up to the value at which the fiber begins to fracture. If the matrix is more ductile than the fibers, then the ultimate tensile strength of the lamina in equation (2) will be transformed to:

$$
\sigma_{1}^{\mathrm{u}}=\sigma_{f}^{u} V_{f}+\sigma_{m}^{f}\left(1-V_{f}\right)
$$

Where the superscript $u$ denotes an ultimate value, and $\sigma_{m}^{f}$ is the matrix stress when the fibers fracture as shown in Figure 2.

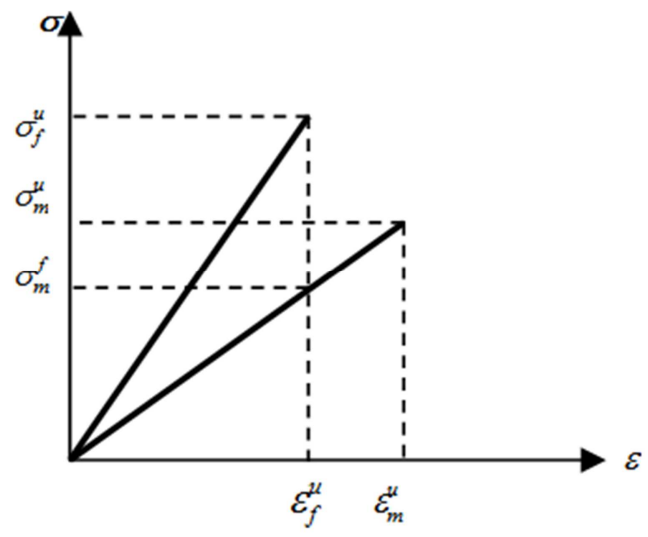

Figure 2. Stress-strain relationships for fiber and matrix.

It is clear that if the fiber volume fraction is very small, the behavior of the lamina is controlled by the matrix.

This can be expressed mathematically as follows:

$$
\sigma_{l}^{u}=\sigma_{m}^{u}\left(1-V_{f}\right)
$$

If the lamina is assumed to be useful in practical applications, then there is a minimum fiber volume fraction that must be added to the matrix. This value is obtained by equating equations (4) and (5) i.e.

$$
V_{\min }=\frac{\sigma_{m}^{u} \sigma_{m}^{f}}{\sigma_{f}^{u}+\sigma_{m}^{u}-\sigma_{m}^{f}}
$$

The variation of the strength of the lamina with the fiber volume fraction is illustrated in Figure 3. It is obvious that when $0<V_{\mathrm{f}}<V_{\text {min }}$ the strength of the lamina is dominated by the matrix deformation which is less than the matrix strength. But when the fiber volume fraction exceeds a critical value (i.e. Vf $>$ VCritical ), Then the lamina gains some strength due to the fiber reinforcement.

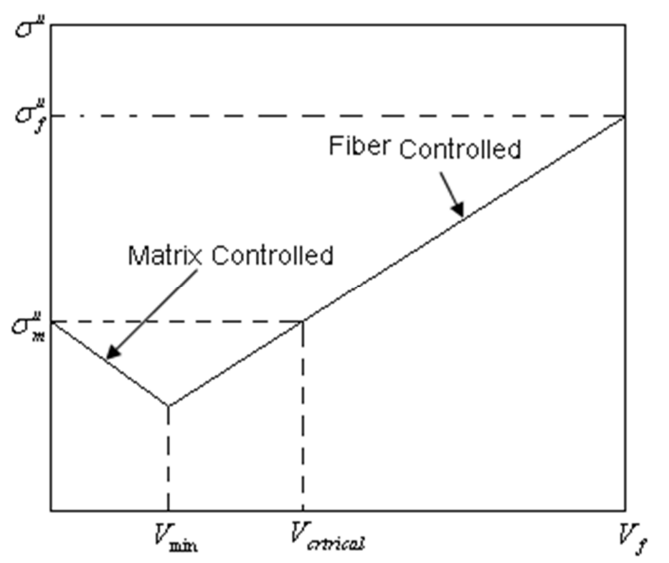

Figure 3. Variation of unidirectional lamina strength with the fiber volume fraction. 
The micromechanical approach is not responsible for the many defects which may arise in fibers, matrix, or lamina due to their manufacturing. These defects, if they exist include misalignment of fibers, cracks in matrix, non-uniform distribution of the fibers in the matrix, voids in fibers and matrix, delaminated regions, and initial stresses in the lamina as a result of its manufacture and further treatment. The above-mentioned defects tend to propagate as the lamina is loaded causing an accelerated rate of failure. The experimental and theoretical results in this case tend to differ. Hence, due to the limitations necessary in the idealization of the lamina components, the properties estimated on the basis of micromechanics should be proved experimentally. The proof includes a very simple physical test in which the lamina is considered homogeneous and orthotropic. In this test, the ultimate strength and modulus of elasticity in a direction parallel to the fiber direction can be determined experimentally by loading the lamina longitudinally. When the test results are plotted, as in Figure 4 below, the required properties may be evaluated as follows: -

$$
E_{1}=\sigma_{1} / \varepsilon_{1} ; \sigma^{u}=P^{u} / A \quad ; \quad v_{12}=-\varepsilon_{2} / \varepsilon_{1}
$$

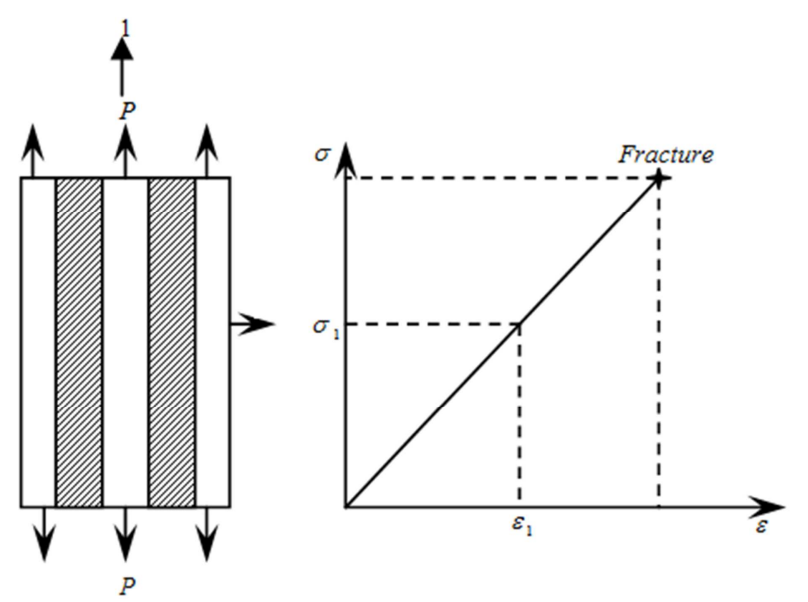

Figure 4. Unidirectional lamina loaded in the fiber-direction.

Similarly, the properties of the lamina in a direction perpendicular to the fiber direction can be evaluated in the same procedure.

\subsection{Analytical Modeling of Composite Laminates}

The properties of a composite laminate depend on the geometrical arrangement and the properties of its constituents. The exact analysis of such structure-property relationship is rather complex because of many variables involved. Therefore, a few simplifying assumptions regarding the structural details and the state of stress within the composite have been introduced.

It has been observed, that the concept of representative volume element and the selection of appropriate boundary conditions are very important in the discussion of micromechanics. The composite stress and strain are defined as the volume averages of the stress and strain fields, respectively, within the representative volume element. By finding relations between the composite stresses and the composite strains in terms of the constituent properties, expressions for the composite moduli could be derived. In addition, it has been shown that, the results of advanced methods can be put in a form similar to the rule of mixtures equations.

Prediction of composite strengths is rather difficult because there are many unknown variables and also because failure critically depends on defects. However, the effects of constituents including fiber-matrix interface on composite strengths can be qualitatively explained. Certainly, failure modes can change depending on the material combinations. Thus, an analytical model developed for one material combination cannot be expected to work for a different one. Ideally a truly analytical model will be applicable to material combination. However, such an analytical model is not available at present. Therefore, it has been chosen to provide models each of which is applicable only to a known failure mode. Yet, they can explain many of the effects of the constituents. (Refer to Ref. [2]).

\section{Literature Review}

Delamination is important phenomenon damage in the laminated composite materials due to weakness of reinforcement through the thickness. The study of the delamination of a laminate may be performed using an approach of fracture mechanics or by introducing appropriate constitutive laws of the interface between the layers constituting the laminate. From a physical point of view, it is reasonable to assume that the second approach can be related to fracture mechanics. In fact, when decohesion occurs between adjacent layers, there is evolution of delaminated surface which is equivalent to the propagation of a crack in a direction a priori known. The literature dealing with the phenomenon of delaminationis very large. A presentation of several structures subjected to the phenomenon of delamination, can be found in $[6,7]$. The delamination phenomenon can be caused by concentration of interlaminar stresses that occur in the vicinity of the free edges or in around of the holes in laminated plates [8]. In addition, the interlaminar defects can grow under a compressive loading. In this case the thin laminated layers degrade (deboning interfaces) and are responsible for increased stresses in the vicinity of the boundaries of delaminated surfaces. In the analysis of the delamination can be distinguished the stage of the crack initiation from the phase of the crack propagation. For prediction of the initiation of a crack from a free edge, the technical Cal culations of the effect of the edge of elasticity [9, $10]$, related to criteria based on the average of the normal stresses on a characteristic distance from the edge of the structure [11] are usually used in post- processor of an elastic design in laminated structures. Delamination does not occur necessarily where the stresses are highest. In the phase of the propagation of a delamination established, approaches based on the linear fracture mechanics are generally used. The rate of energy release $\mathrm{G}$ is a parameter that is often used to describe 
the behavior of the phenomenon of delamination in composite materials and structures. $G$ is defined as the energy released from the newly fractured surface and compared to the critical value Gc (This method is used by many authors for the study of crack propagation $[12,13]$, but not treat the problem of the initiation of a delamination crack. In contrary the approach of the damage mechanics of the composite can describe the initiation of delamination. The rate of energy release is calculated from the forces and nodal displacements [14] and [15]. The state equations and the evolution laws of the interface provided in the context of thermodynamics are described in [16-22]. Models of elastic and damageable interface are presented in [23-27] and generalized in [28]. In these models, special interface elements are applied in areas where the delamination phenomenon is likely to occur. Elements plane strain with cubic interpolation functions were introduced for discretization of the laminate ply [29]. Other models have been developed for modeling damage layer [30] and interface phenomena [31]. These models are based on the damage mechanics. The interface is considered as a three-dimensional medium with negligible thickness compared to the other dimensions. Therefore, the interface can be considered as two-dimensional entity witch transfers traction and displacement from one layer to the other [32]. The interface is assumed to be dependent on the fiber orientation of adjacent layers and it is assumed to be elastic and damageable. Delamination may be caused by interlaminar stress [9]. The objective of this paper is to present a method to simulate progressive delamination based on a new mixed-mode failure criterion in the context of damage mechanics. This study will highlight the positive contribution of the powder core dates incorporated in the new woven composite. The date cores powder incorporation has an increase effect of the mechanical characteristics giving to the hybrid composite a better behavior and reducing certain types of degradation like delamination.

It is important to recognize that, with the advent of composite media, certain new material imperfections can be found in composite structures in addition to the better-known imperfections that one finds in metallic structures. Thus, broken fibers, delaminated regions, cracks in the matrix material, as well as holes, foreign inclusions and small voids constitute material and structural imperfections that can exist in composite structures. Imperfections have always existed and their effect on the structural response of a system has been very significant in many cases. These imperfections can be classified into two broad categories: initial geometrical imperfections and material or constructional imperfections.

The first category includes geometrical imperfections in the structural configuration (such as a local out of roundness of a circular cylindrical shell, which makes the cylindrical shell non-circular; a small initial curvature in a flat plate or rod, which makes the structure non-flat, etc.), as well as imperfections in the loading mechanisms (such as load eccentricities; an axially loaded column is loaded at one end in such a manner that a bending moment exists at that end). The effect of these imperfections on the response of structural systems has been investigated by many researchers and the result of these efforts can be easily found in books [3], as well as in published papers [33-50].

The second class of imperfections is equally important, but has not received as much attentions as the first class; especially as far as its effect on the buckling response characteristics is concerned. For metallic materials, one can find several studies which deal with the effect of material imperfections on the fatigue life of the structural component. Moreover, there exist a number of investigations that deal with the effect of cut-outs and holes on the stress and deformation response of thin plates. Another material imperfection is the rigid inclusion. The effect of rigid inclusions on the stress field of the medium in the neighborhood of the inclusion has received limited attention. The interested reader is referred to the bibliography of Professor Naruoka [33].

There exist two important classes of material and constructional-type imperfections, which are very important in the safe design, especially of aircraft and spacecraft. These classes consist of fatigue cracks or cracks in general and delamination in systems that employ laminates (i.e. fiberreinforced composites). There is considerable work in the area of stress concentration at crack tips and crack propagation. Very few investigations are cited, herein, for the sake of brevity. These include primarily those dealing with plates and shells and non-isotropic construction. Some deal with cracks in metallic plates and shells [51-54]. Others deal with nonisotropic construction and investigate the effects of nonisotropy [55-60]. In all of these studies, there is no mention of the effect of the crack presence on the overall stability or instability of the system.

Finally, delamination is one of the most commonly found defects in laminated structural components. Most of the work found in the literature deals with flat configurations.

Composite structures often contain delamination. Causes of delamination are many and include tool drops, bird strikes, runway debris hits and manufacturing defects. Moreover, in some cases, especially in the vicinity of holes or close to edges in general, delamination starts because of the development of interlaminar stresses. Several analyses have been reported on the subject of edge delamination and its importance in the design of laminated structures. A few of these works are cited [61-67]. These and their cited references form a good basis for the interested reader. The type of delamination that comprises the basic and primary treatise is the one that is found to be present away from the edges (internal). This delaminating could be present before the laminate is loaded or it could develop after loading because of foreign body (birds, micrometer, and debris) impact. This is an extremely important problem especially for laminated structures that are subject to destabilizing loads (loads that can induce instability in the structure and possibly cause growth of the delamination; both of these phenomena contribute to failure of the laminate). The presence of delamination in these situations may cause local buckling and / or trigger global buckling and therefore induce a reduction in the overall load-bearing capacity of the laminated structure. The problem, because of its importance, 
has received considerable attention.

\section{Conclusions}

Delamination is a critical failure mode in fiber-reinforced composite decks plates and beams. It may lead directly to through-thickness failure owing to interlaminar stresses caused by out of plane loading, curved or tapered geometry, or discontinuities owing to cracks, ply drops or free edges. Impact loading causes multiple delaminations, which can propagate in conjunction with sub laminate buckling, greatly reducing the residual compressive strength.

\section{References}

[1] David Roylance, ' an introduction to composite materials', Department of material science and engineering, Massachusetts Institute of Technology, Cambridge; (2000).

[2] Stephen W. Tsai, Thomas Hahn H., ' introduction to composite materials', Technomic publishing company; (1980).

[3] Turvey G. J., Marshall I. H., 'buckling and post buckling of composite plates', Great Britain, T. J. press Ltd, Padstow, Cornwall; (1995).

[4] Vernon B. John, ' introduction to engineering materials', second edition; (1972).

[5] Jan Stegmann and Erik Lund, ' notes on structural analysis of composite shell structures', Aalborg University, Denmark; (2001).

[6] A. C. Garg, A damage model in composite structures, Engineering Fracture Mech., 1998. vol. 29.

[7] O. O. Ochoa. and Reddy J. N, Finite elements analysis of composites laminates, Kluwer, 1992.

[8] S. Jain and D. C. H. Yang, Effects of federate and chisel edge on delamination in composite drilling. Processing and Manufacturing of Composite Materials, ASME PED 49. 1991. Vol. 27.

[9] D. Engrand, Boundary layer approach to the Calculation of Transverse stresses along the Free Edge of a Symmetric Laminated Plate of Arbitrary Width under in Plane Loading, Composites Structures, 1981.

[10] H. Dumontet., Study of Boundary layer Problem in Elastic composite Materials, M2AN, 20, 1986.

[11] R. Y. Kim. and S. R. Sony, Delamination of Composite Laminates Stimulated by interlaminar Shear, ASTM-STP 893, 1986.

[12] A. S. D. Wang, M. Slomania. and R. B. Bucinell, Delamination Crack Growth in Composite Laminates. Delamination and Debonding of Materials, JOHNSON W. S (Ed.), ASTMSTP, 1985.

[13] A. S. D. WANG, Fracture Analysis of Interlaminar cracking, Interlaminar response of Composite Materials, Composite materials Series, PAGANO N. J. (Ed.) Elsevier, 1989.

[14] D. B. Davidson., R. Kruger and M. König. Effect of stacking sequence on energy release rate distribution in multi-directional DCB and ENF specimens. Engineering Fracture Mech., 1996. vol. 55-4.

[15] T. K. O'Brien, Mixed-mode strain-energy-release rate effects on edge delamination of composites, In: damage in composite Materials, ASTM STP 836, ASTM, Philadelphia, PA, 1984.

[16] M. Fremond. Adherence des solides, J. Mécan. Théor. Appl. 6, 1987.

[17] M. Fremond. Contact with Adhesion. In Topic in Non-smooth Mechanics, Birkhäuser, 1988.

[18] N. Point, Approche Mathématiques de problèmes à frontières libres: Application à des exemples physiques, Thèse de doctorat d'Etat Es-Sciences Mathématiques de l'Université Paris XIII, 1989.

[19] J. M. Truong Dinh Tien, Contact avec adhérence. Thèse de Doctorat de l’Université Paris VI, 1990.

[20] L. Ascione and D, Bruno On delamination problem of two layer plates. In unilateral problems in Structural Analysis, Springer, Berlin, 1985.

[21] A. Girmaldi and J. N, Reddy On delamination in plates: a unilateral contact approach. In Unilateral Problems in Structural Analysis, Springer, Berlin, 1985.

[22] PLadevèze. A damage computational method for composite structures. Computer Structures, (1992). vol. 44.

[23] O. Allix. and P. Ladevèze, Interlaminar interface modeling for the prediction of delamination, Composites Structures, 1992.

[24] A. Corigliano, Formulation identification and use of interface models in the numerical analysis of composite delamination, Int. J. Solids Structures, 1993. vol. 30.

[25] J. C. Schellekens and DE R. Borst, Free edge delamination in carbon epoxy laminates: A novel numerical/experimental approach, Composites Structures, 1993. vol. 28.

[26] Leandro José da Silva1, TúlioHallak Panzera, André Luis Christoforo, Luís Miguel Pereira Durão, Francisco Antonio Rocco Lahr, Numerical and Experimental Analyses of Bio composites Reinforced with Natural Fibers, International Journal of Materials Engineering 2012, 2 (4): 43-49.

[27] D. Benzerga, A. Haddi., ASeddak. and ALavie, mixed-mode damage model for delamination growth applied to a new woven composite, Computational Materials Science, 2008. vol. 41, pp. 515-521.

[28] P. Ladevèze and E. Ledantec, Damage modeling of the elementary ply for laminated composites, Composites Sciences and technology, 1992. vol. 43.

[29] O. Alix and P. Ladevèze, Interlaminar Interface modeling for Prediction of Delamination, Composites Structures, 1992. vol. 22.

[30] F. Gruttmann and W. Wagner, On the numerical analysis of local effects, Composites structures, 1994. vol. 29.

[31] J. C. Simo and M. S. Rifai. A, class of mixed assumed strain methods and the method of incompatible modes, Int. Num. Meth. Eng, 1990. vol. 29.

[32] P. P. Camanho, C. G. Dàvila and D. R Ambur, Numerical Simulation of Delamination Growth in Composite Materials. NASA/TP -211041, 2001. 
[33] Naruoka M., 'Bibliography on theory of plates', Gihodo, Tokyo; (1981).

[34] Winterstetter Th. A. and Schmidt H., 'Stability of circular cylindrical steel shells under combined loading', Thin-walled structures; (2002), 40: PP. (893-909).

[35] Pircher M., and Bridge R., ' The influence of circumferential weldinduced imperfections on the buckling of silos and tanks', Journal of constructional steel research; (2001), 57 (5): PP. (569-580).

[36] Deml M., and Wunderlich W.,' Direct evaluation of the worst imperfection shape in shell buckling', Computer methods in applied mechanics and engineering; (1997), 149 [1-4]: PP. (201-222).

[37] Arbocz J., and Starnes J. H.,' Future directions and challenges in shell stability analysis', Thin-walled structures; (2002), 40: PP. (729-754).

[38] Arbocz J.,' The effect of imperfect boundary conditions on the collapse behavior of anisotropic shells', International Journal of solids and structures; (2000), 37: PP. (6891-6915).

[39] Huhne C., Zimmermann R., Rolfes R., and Geier B., 'loading imperfections-experiments and Computations', Euromech Colloquium 424; (2001), the Netherlands.

[40] Geier B., Klein H., and Zimmermann R.,' Buckling tests with axially Compressed unstiffened cylindrical shells made from CFRP', proceedings, International Colloquium on buckling of shell structures on land, in the sea, and in the air, Elsevier applied sciences; (1991), London and New York: PP. (498-507).

[41] Geier B., Klein H., and Zimmermann,' Experiments on buckling of CFRP Cylindrical shells under non-uniform axial load', proceedings of international conference on Composites engineering; (1994).

[42] Albus J., Gomez-Garcia J., and oery H.,' Control of assembly induced stresses and deformations due to the waviness of the interface flanges of the ESC-An upper stage', 52nd International astronautical congress; (2001), Toulouse, France.

[43] Zimmermann R.,' Buckling research for imperfection tolerant fiber composite structures', proceeding of the conference on spacecraft structures, material and mechanical testing, Nordwijk, The Netherlands: (1996).

[44] Meyer-Piening H. R., Farshad M., Geier B., and Zimmermann, 'Buckling loads of CFRP Composite cylinders under combined axial and torsion loading-experiments and computations', Composite structures; (2001), 52: PP. (427-435).

[45] CMH-17 (Composite Material Handbook-17), Material science corporation; (2010).

[46] Guo S. J.,' Stress concentration and buckling behaviour of shear loaded composite panels with reinforced cutouts', Composite structures;( 2007): PP. (1-9).

[47] Remmers J. J. C., and de Borst R.,' Delamination buckling of fiber-metal laminates', Composite science and technology; (2001): PP. (2207-2213).

[48] Vit obdrzalek, and Jan Vrbka,' Buckling and post buckling of a large delaminated plate subjected to shear loading', Engineering mechanics, vol. 16; (2009), No. 4: PP. (297-312).

[49] Vit obdrzalek, and Jan Vrbka,' On buckling of a plate with multiple delaminations', Engineering mechanics, vol. 17; (2010), No. 1: PP. (37-47).
[50] Keiichi Nemoto, Hirakazu Kasuya, Hisao Kikugawa, and Takashi Asaka,' post buckling behavior of composite laminated plates with initial imperfections under biaxial compression', Materials transactions, vol. 50, No. 2; (2009): PP. (299-304).

[51] Takao Y., Taya M., and Chou T. W.,' Stress field due to cylindrical inclusion with constant axial Eigen strain in an infinite elastic body', Journal of applied mechanics; (1981), 48 (4): PP. (853-858).

[52] Lakshminarayana H. V., and Murthy M. V. V.,' On stresses around an arbitrarily oriented Crack in cylindrical shell', International Journal of fracture; (1976), 12 (4): PP. (547-566).

[53] Twee J., and Rooke D. P,' The stress intensity factor for a crack at the edge of a loaded hole', International Journal of solids and structures; (1979), 15: PP. (899-906).

[54] Dyshel M. S.,' Fracture of plates with cracks under tension after loss of stability', Journal of applied mathematics and mechanics (PMM); (1981), 17 (4): PP. (77-83).

[55] Erdogan F., Ratwani M., and Yuceoglu U.,' On the effect of orthotropy in cracked cylindrical plates', International Journal of fracture; (1974), 10 (4): PP. (369-374).

[56] Krenk S.,' Influence of transverse shear on an axial crack in a cylindrical shell', International Journal of fracture; (1978), 14 (2): PP. (123-142).

[57] Delale F., and Erdogan F.,' Effect of transverse shear and material orthotropy in a cracked spherical cap', International Journal of solids and structures; (1979), 15: PP. (907-926).

[58] Lakshminarayana H. V., and Murthy M. V. V,' On a finite element model for the analysis of through cracks in laminated anisotropic cylindrical shells', Engineering fracture mechanics; (1981), 14 (4): PP. (697-712).

[59] Theocaris P. S., and Milios J.,' Crack-arrest at a bimaterial interface', International Journal of solids and structures; (1981), 17: PP. (217-230).

[60] Rogers T. G.,' Crack extension and energy release rates in finitely deformed sheet reinforced with inextensible fibers', International Journal of solids and structures; (1982), 18: PP. (705-721).

[61] Kachanov L. M.,' Separation failure of composite materials', polymer mechanics; (1976), 6 (12): PP. (812-815).

[62] Williams J. G., and et al.,' Recent developments in the design, testing and impact damage-tolerance of stiffened composite plates', Nasa TM 80077; (1979), April.

[63] Wilkins D. J, and et al.,' characterizing delamination growth in graphite-epoxy', Damage in composite materials; (1982): PP. (168-183).

[64] Wang S. S.,' Edge delamination in angle-ply composite laminates', AIAA Journal; (1984), 22 (2): PP. (256-264).

[65] Scott W. Beckwith,' Manufacturing defects in composite structures', Sample Journal, volume 48, No. 5; September/October (2012).

[66] M. Vable,' Stability of columns', Mechanics of materials; (2014): chapter eleven: PP. (496-528).

[67] CPNI EBP,' Influence of delamination of laminated glass on its blast performance', July (2013). 


\section{Biography}

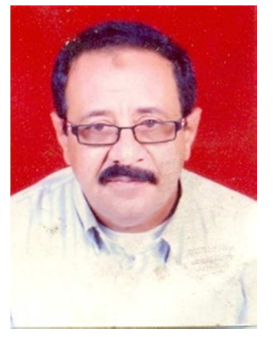

Osama Mohammed Elmardi Suleiman Khayal was born in Atbara, Sudan in 1966. He received a bachelor degree in mechanical engineering from Sudan University of science and technology-Faculty of engineering in 1998, and a master degree in solid mechanics from Nile valley university (Atbara, Sudan) in 2003, and a PhD in structural engineering in 2017. The author also works as a technical manager and superintendent of Al-Kamali mechanical and production workshops group which specializes in small, medium and large automotive overhaul maintenance and which situated in Atbara town in the north part of Sudan, River Nile State. 